\title{
Can the environmental TEM confirm atomistic models of adsorbed molecules at surfaces of solids?
}

\author{
Matthieu Bugnet ${ }^{1}$, Ihor Pershukov ${ }^{1}$, Olivier Matz ${ }^{2}$, Monica Calatayud ${ }^{2}$, Thierry Epicier ${ }^{1}$ \\ 1. Univ Lyon, INSA Lyon, UCBL Lyon1, MATEIS, CNRS UMR 5510, F-69621 Villeurbanne, France \\ 2. Sorbonne Universités, UPMC Univ Paris 06, CNRS, Laboratoire de Chimie Théorique, 75252 Paris \\ Cedex 05, France
}

It is well established that enhanced atomic mobility exists at ceria $\left(\mathrm{CeO}_{2}\right)$ surfaces in high vacuum (HV) conditions within the TEM, owing to beam-induced reduction effects $[1,2]$. Controlling the atmosphere surrounding the specimen within an aberration-corrected environmental TEM (ETEM) allows to monitor the atomic mobility at $\{100\}$ surfaces, and to directly visualize the projected atomic structure of the top surface of ceria nanocubes through high resolution TEM (HRTEM) imaging [1]. Therefore, it is pertinent to investigate the current capabilities of the ETEM to validate atomistic models of adsorbed molecules at the surface of materials. However, it is only possible to conclude about the nature of the contrasts at the surface by comparing the experimental HRTEM images to image simulations. In 2012, Yoshida et al. demonstrated the presence of $\mathrm{CO}$ molecules adsorbed on pure gold nanoparticles [3] using this approach. In the present work, we build on the prospect of directly observing light elements resulting from the adsorption of $\mathrm{CO}_{2}$ molecules at the surface of a highly challenging material in transmission electron microscopy, $\mathrm{CeO}_{2}$. Indeed, ceria is of high interest for research in catalysis [4] and pharmaceuticals [5], however it reduces strongly and spontaneously under the electron beam in HV, and the intrinsic presence of a light element like oxygen complicates significantly quantitative high resolution imaging.

Here, HRTEM is carried out in a FEI Titan ETEM G2 80-300. This instrument is equipped with a Csaberration corrector of the image-forming lens, a CMOS detector GATAN Oneview capable of fast recording ( $25 \mathrm{fps}$ in $4 \mathrm{k} \times 4 \mathrm{k}$ ), and a high stability DENSSolutions Wildfire ST holder for high temperature experiments. The structural models of $\{100\}$ surfaces with oxygen coverage, or with adsorbed $\mathrm{CO}_{2}$ molecules, are obtained within the DFT framework [6]. The HRTEM image simulations were performed using the JEMS software.

In an oxygen-rich atmosphere, which consists here of pure $\mathrm{O}_{2}$ introduced in the ETEM, the $\{100\}$ surface termination can be observed directly, as shown in Figure 1. White contrast of atomic columns was chosen here, as it is well known to provide high contrast of oxygen in $\mathrm{CeO}_{2}$ [2]. The image simulation fits reasonably well the experimental contrast observed at the surface, for both the top surface $\mathrm{O}$-atoms, and the Ce atomic plane located just below. It is also noticeable that about two unit cell planes below the surface in the bulk part of ceria, the O columns appear larger than Ce columns, as confirmed in the simulation. This observation, as well as small discrepancies between experimental and simulated images, are likely due to dynamical effects and possible residual misalignments. Also, variations of the contrast at the surface arising from defects, such as surface steps or terraces as pointed out in Figure 1, will be discussed. Figure 2 shows a HRTEM image of $\mathrm{CO}_{2}$ adsorbed at the $\{100\}$ surface. Clearly, the contrast at the top surface is more elongated parallel to the surface than in Figure 1, when oxygen is adsorbed at the surface. This peculiar contrast is attributed to the presence of surface carbonates or $\mathrm{CO}_{3-}$ based complexes as it will also be discussed on the basis of image simulations. The effect of temperature on the variation of this contrast will be presented in terms of surface desorption.

In conclusion, the present study demonstrates the direct visualization of adsorbed oxygen or $\mathrm{CO}_{2}$ molecules at $\{100\}$ surfaces of ceria. The nature of the contrasts is evaluated from HRTEM simulations, 
which reproduce quantitatively the experimental HRTEM images, on the basis of DFT-calculated adsorption models. This work highlights the potential of environmental TEM at high spatial resolution to directly image adsorbed molecules, and thereby better understand the surface coverage and the nature of adsorption sites predicted by atomistic calculations [7].

References:

[1] M. Bugnet et al., Nano Letters 17, $7652-7658$ (2017).

[2] Y. Lin et al., Nano Letters 14, 191-196 (2014).

[3] H. Yoshida et al., Science, 335 (6066), 317-319 (2012).

[4] A. M. Glänzer et al., Angewandte Chemie International Edition, 56(42), 13078-13082 (2017).

[5] I. Trenque et al., Phys Chem. Chem. Phys. (2019), DOI:10.1039/c9cp00179d.

[6] O. Matz and M. Calatayud, ACS Omega 3, 11, 16063-16073 (2018).

[7] M. B. acknowledges funding from INSA Lyon through a BQR project THERMOS. The authors are grateful to $\mathrm{M}$. Aouine for assistance in TEM preparation. Z. Wu from ORNL, USA is gratefully acknowledged for providing ceria nanocubes. The electron microscopy work presented here has been performed on a FEI Titan ETEM at the Lyon-St-Etienne centre for electron microscopy (www.clym.fr).

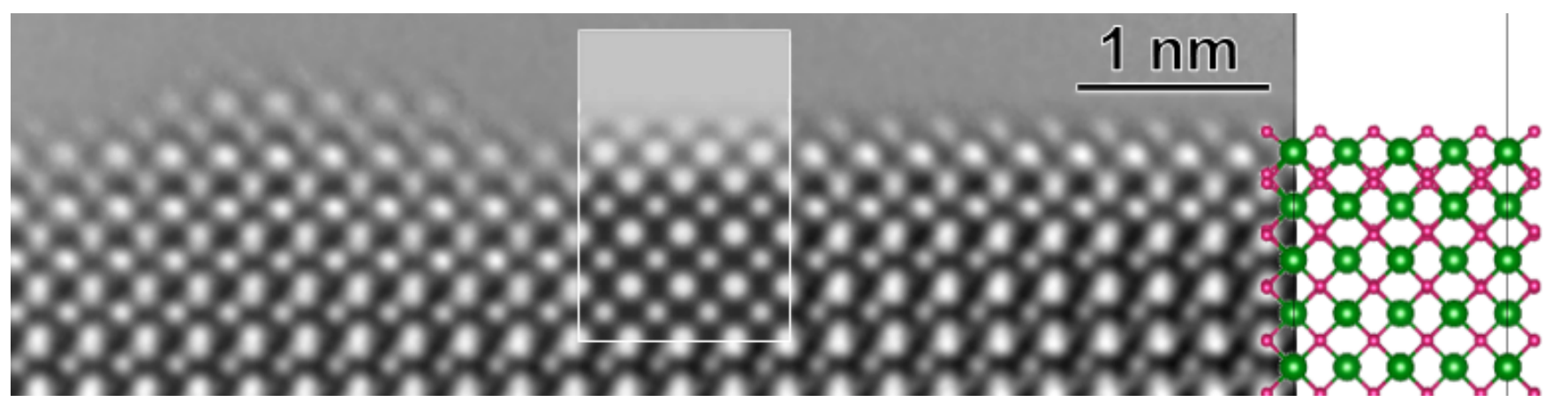

Figure 1. Experimental $\mathrm{HR}(\mathrm{E}) \mathrm{TEM}[001]-\mathrm{CeO}_{2}$ image in 'white atom' contrast acquired under environmental conditions $\left(\mathrm{P}\left(\mathrm{O}_{2}\right)=510^{-2}\right.$ mbar). The image shown was obtained after realignment of a stack of $2504 \mathrm{kx} 4 \mathrm{k}$ frames recorded at $12 \mathrm{fps}$ and averaged to improve the SNR. The simulation (inset) was calculated at a defocus of $10 \mathrm{~nm}$ and a thickness of $3.3 \mathrm{~nm}$ (6-cells thick area) from the DFT model shown on the right with a fully occupied oxygen (smaller dots) terminating layer (stable configuration). Similar uniform contrasts are observed all along the surface even on the terrace visible on the left.

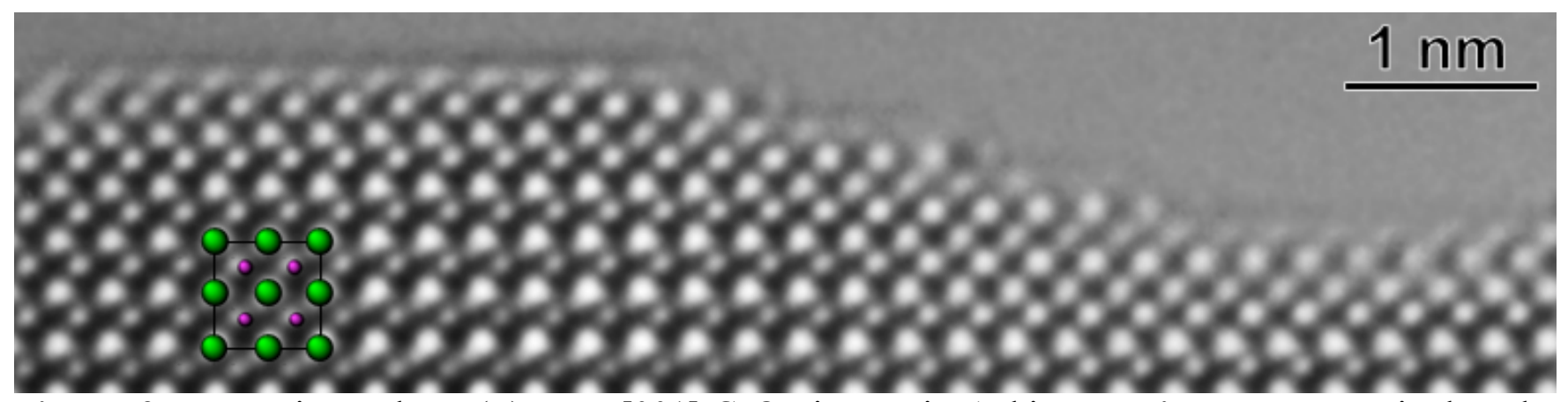

Figure 2. Experimental $\mathrm{HR}(\mathrm{E}) \mathrm{TEM}[001]-\mathrm{CeO}_{2}$ image in 'white atom' contrast acquired under environmental conditions $\left(\mathrm{P}\left(\mathrm{CO}_{2}\right)=710^{-1} \mathrm{mbar}\right)$. The image shown was obtained after realignment of a stack of $1004 \mathrm{kx} 4 \mathrm{k}$ frames recorded at $10 \mathrm{fps}$ and averaged to improve the SNR. Inset: Ce(large green dots); $\mathrm{O}$ (small purple dots). 